\title{
Variations in the gonadal artery with a single common trunk: embryological hypotheses by observation
}

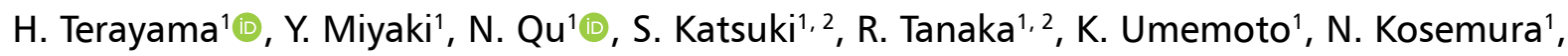 \\ K. Suyama', O. Tanaka', K. Sakabe ${ }^{1}$
}

'Department of Anatomy, Division of Basic Medicine, Tokai University School of Medicine, Kanagawa, Japan ${ }^{2}$ Department of Rehabilitation, Kanto Rosai Hospital, 1-1 Kizuki Sumiyoshi-cho, Nakahara-ku, Kawasaki-si, Kanagawa, Japan

[Received: 5 March 2020; Accepted: 31 March 2020]

Background: A gonadal artery originates as a branch of the abdominal aorta and renal artery inferior to the level of origin of the renal arteries. Variations in multiple right testicular arteries (RTAs) arising from the abdominal aorta are common. We aimed to re-evaluate the unusual courses of gonadal arteries with a single common trunk in relation to the inferior vena cava and left renal vein and explain the developmental anatomy.

Materials and methods: The observational cross-sectional study was performed on 54 Japanese adult cadavers (29 men and 25 women). We examined the literature and developed embryological hypotheses on the single common trunk of the gonadal artery.

Results: The gonadal artery, testicular artery, and ovarian artery arose from the abdominal aorta in $93.1 \%, 96.3 \%$, and $89.6 \%$ of cases, respectively, and from the renal artery in $4.9 \%, 3.7 \%$, and $6.3 \%$ of cases, respectively. We found two rare variations in the RTAs observed during the routine dissection of two male cadavers; in these two cases, a single common trunk of the RTAs originated from the abdominal aorta. A single common trunk was found in 3.7\% of cadavers, $2.0 \%$ of sides, and $2.0 \%$ of arteries in the gonadal artery and in $6.9 \%$ of cadavers, $3.8 \%$ of sides, and $3.7 \%$ of arteries in the testicular artery. All cases of the single common trunk, including those in past reports, were observed only in men.

Conclusions: Knowledge of the variations in RTAs has important clinical consequences for invasive and non-invasive arterial procedures. In addition, this variation provides a new interpretation of the embryology of the gonadal artery. Variations similar to our findings have not been previously reported. Therefore, different variations concerning the RTA should be considered during surgical and non-surgical evaluations. (Folia Morphol 2021; 80, 2: 324-330)

Key words: single common trunk, right testicular artery, vascular development, cadaver 


\section{INTRODUCTION}

The gonadal artery (GA) originates separately on the left and right sides as a lateral branch of the abdominal aorta (AA), inferior to the level of origin of the renal arteries and veins. On the right side, the artery commonly passes anterior to the inferior vena cava (IVC), while on the left, it passes posterior to the inferior mesenteric vein. It descends inferolaterally toward the pelvis along the psoas major muscle, under the parietal peritoneum, and then enters into the ipsilateral deep inguinal ring [11].

Variations of the renal, adrenal, and inferior phrenic arteries, among others, are reported to occur at the origin of the GA as well as at the AA [1, 5, 7-9, 12]. In addition, variations of the courses of multiple GAs are reported $[1,5-10,12,15,17]$. Even the occurrence of many GAs with multiple origins has been discussed. Multiple GAs have a single origin, and the multiple branches pass through several blood vessels.

The course of GAs is important not only for identifying the structures of the gonadal anatomy but also for facilitating safer gonadal and renal surgeries. It is unclear whether the single common trunk can be included for multiple arteries or single arteries, and no study so far has focused on GAs with a single common trunk. In addition, the embryological considerations for the development of GAs with a single common trunk have not been reported.

In the present study, we re-evaluated the unusual courses of GAs with a single common trunk with respect to the IVC and left renal vein and attempted to explain the developmental anatomy.

\section{MATERIALS AND METHODS}

All cadavers were selected from the bodies used for research and anatomy practice at Tokai University School of Medicine in 2017 and 2018. This observational cross-sectional study was performed on 54 Japanese adult cadavers ( 29 men and 25 women) and on 98 sides ( 53 male and 45 female sides) and on 102 arteries (54 testicular and 48 ovarian arteries), with an average age of $88.6 \pm 7.46$ years $(88.0 \pm 6.32$ years in men and $89.1 \pm 8.29$ years in women). Some left and right sides of the cadavers were not observable by human error. In addition, there were cadavers with multiple gonadal arteries. Therefore, the results are presented in terms of cadavers, sides, and arteries. Cadavers that underwent urogenital surgery were excluded from this study. The single common trunks were treated as one artery.
We also presented case results from the dissection of 94-year-old (No. 2026) and 87-year-old (No. 2028) Japanese adult male cadavers as the first and second cases (cause of death: hepatic cancer and pancreatic cancer, respectively).

The cadavers were free from other diseases of the liver, stomach, and related vessels. Gross dissection was performed using the customary procedure. The vascular anatomical relationship around the kidney was specifically observed. For this purpose, we removed the branches of the coeliac trunk and the upper mesenteric arteries and carefully examined the structures.

\section{RESULTS}

The two cadavers with the single common trunk were referred to as first and second cases (Figs. 1 and 2, respectively). In Figure 1, the right testicular arteries (RTAs) of the single common trunk arose from the $A A$ at the second lumbar vertebral level below the level of the left renal vein and ran $4 \mathrm{~mm}$ toward the IVC. Afterward, the single common trunk divided into two testicular arteries (TAs) passing through the lateral (dorsal) side (IRTA) and the medial (ventral) side (mRTA) of the IVC. The IRTA travelled in an arch shape from the dorsal side to the ventral side with respect to the right renal vein and descended to the caudal side. The mRTA passed through the ventral side of the right renal artery and descended to the caudal side. The IRTA and mRTA joined at the inferior pole of the right kidney and descended ventrally to the psoas major muscle. In other words, the IVC passed through the hiatus of the RTA. The left testicular arteries (LTAs) arose from the AA at the second lumbar vertebral level and descended ventrally to the psoas major muscle. The single common trunk of the RTAs, mRTA, IRTA, and LTA had an external diameter of approximately $1.8 \mathrm{~mm}, 0.8 \mathrm{~mm}, 1.8 \mathrm{~mm}$, and $3.6 \mathrm{~mm}$, respectively (Fig. 1). The right renal artery arose from the $A A$ at the third lumbar vertebral level and passed through the ventral side of the IVC. The other abdominal arteries displayed normal courses.

In Figure 2, RTAs of the single common trunk arose from the $A A$ at the third lumbar vertebral level below the level of the left renal vein and then ran $30 \mathrm{~mm}$ toward the IVC in the second case. Afterward, the single common trunk divided into two testicular arteries on the ventral side of the IVC and passed through the IRTA and the MRTA of the right testicular vein. The IRTA and MRTA did not join but dropped 


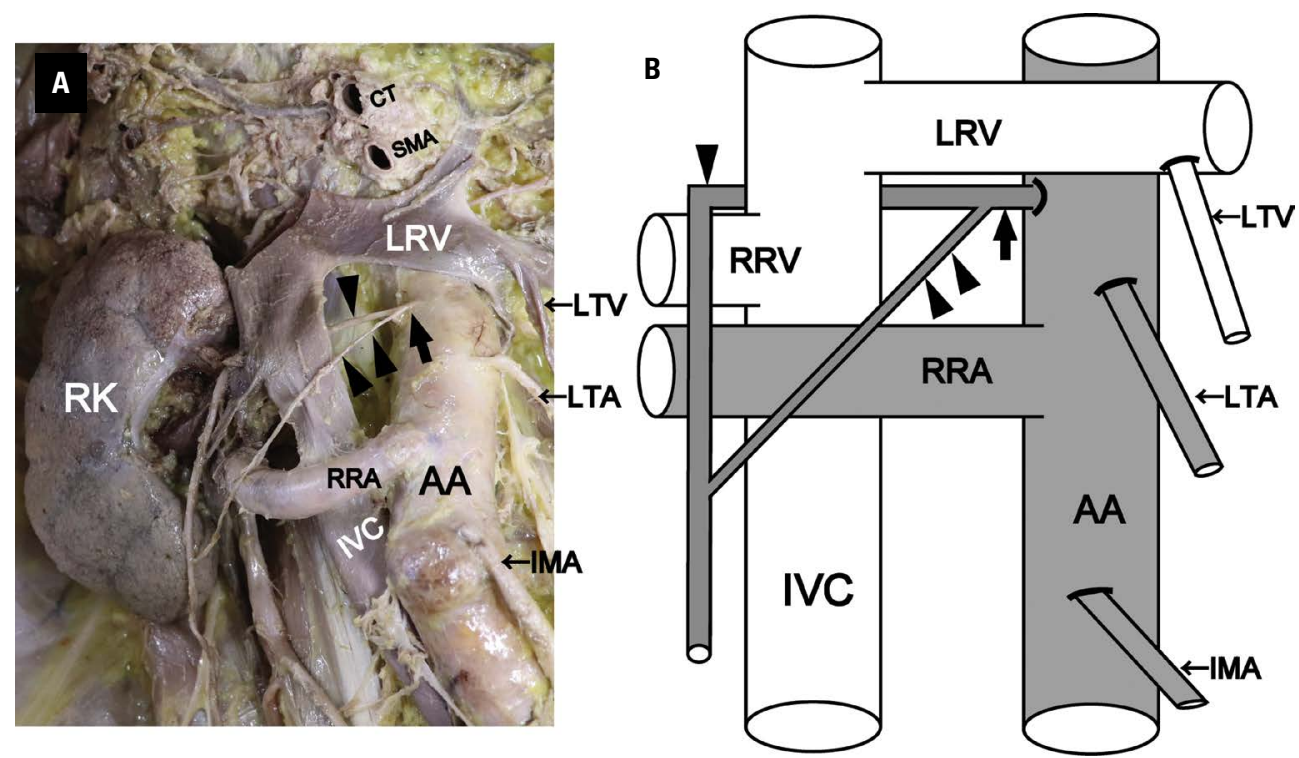

Figure 1. Photograph and schematic of the right testicular artery with the single common trunk in the first cadaver; A. Anterolateral view of a formaldehyde-fixed abdominal cavity of a 94-year-old man (cause of death: hepatic cancer; No. 2026); B. Graphical schematic of the same view. The black arrow points to the single common trunk of the right testicular artery. The single and double black arrowheads point to the lateral right testicular artery and medial testicular artery, respectively; AA — abdominal aorta; CT — coeliac trunk; IMA — inferior mesenteric artery; IVC — inferior vena cava; LRV — left renal vein, LTA — left testicular artery; LTV — left testicular vein; RK — right kidney; RRA — right renal artery; RRV — right renal vein; RTV — right testicular vein; SMA — superior mesenteric artery.
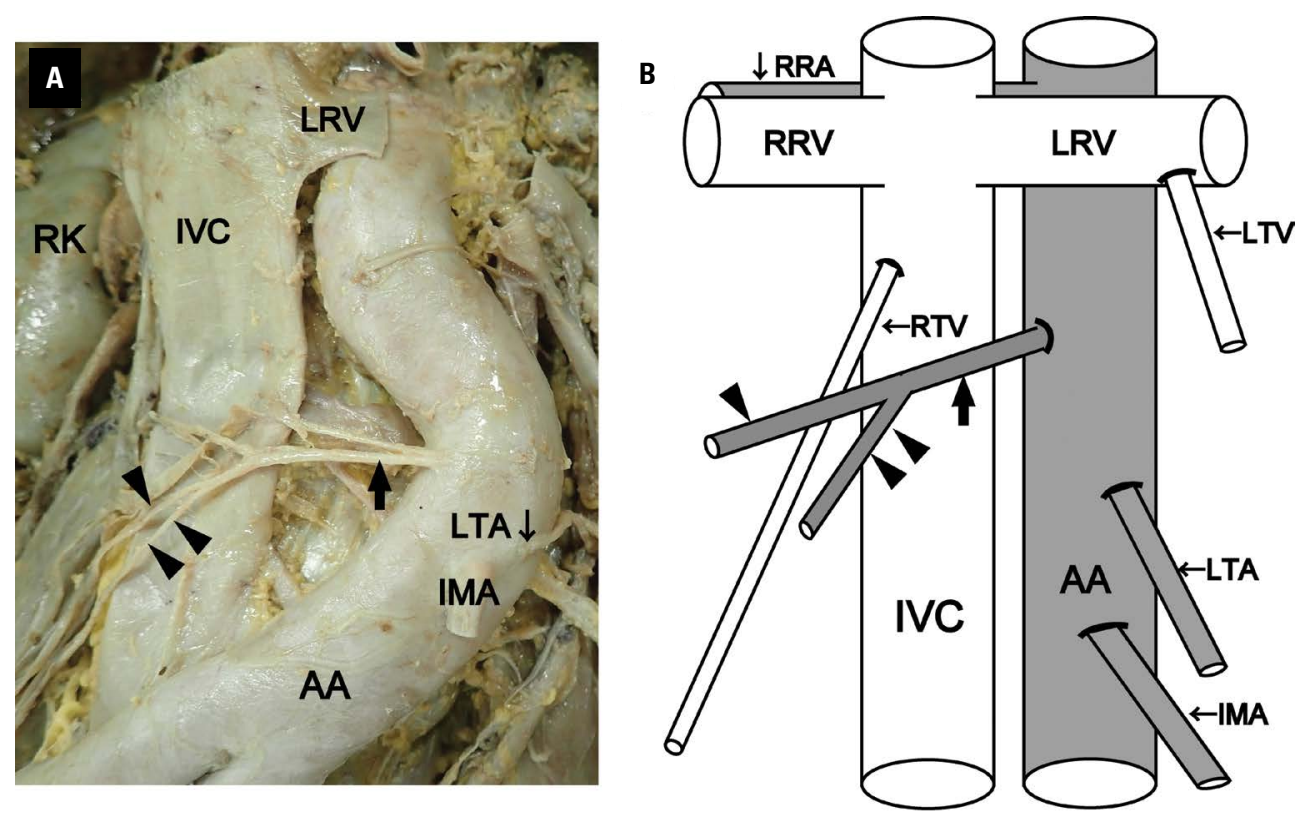

Figure 2. Photograph and schematic illustration of the right testicular artery with the single common trunk in the second cadaver; $\mathbf{A}$. Anterolateral view of a formaldehyde-fixed abdominal cavity of an 87-year-old man (cause of death: pancreatic cancer; No. 2028); B. Graphical schematic of the same view. The black arrow points to the single common trunk of the right testicular artery. The single and double black arrowheads point to the lateral right testicular artery and medial testicular artery, respectively; AA — abdominal aorta; IMA — inferior mesenteric artery; IVC — inferior vena cava; LRV — left renal vein; LTA — left testicular artery; LTV — left testicular vein; RK — right kidney; RRA — right renal artery; RRV — right renal vein.

separately toward the deep inguinal ring. The left testicular arteries arose from the AA at the fourth lumbar vertebral level and descended ventrally to the psoas major muscle. The single common trunk of the 
Table 1. Observed anatomical variations in the origin of gonadal arteries of the present study

\begin{tabular}{|c|c|c|c|c|c|c|c|c|c|}
\hline \multirow{2}{*}{$\begin{array}{l}\text { Origin of go- } \\
\text { nadal artery }\end{array}$} & \multicolumn{3}{|c|}{ Male (testicular artery) } & \multicolumn{3}{|c|}{ Female (ovarian artery) } & \multicolumn{3}{|c|}{ Total (gonadal artery) } \\
\hline & Body & $\begin{array}{c}\text { Side } \\
\text { (left, right) }\end{array}$ & $\begin{array}{c}\text { Artery } \\
\text { (left, right) }\end{array}$ & Body & $\begin{array}{c}\text { Side } \\
\text { (left, right) }\end{array}$ & $\begin{array}{c}\text { Artery } \\
\text { (left, right) }\end{array}$ & Body & $\begin{array}{c}\text { Side } \\
\text { (left, right) }\end{array}$ & $\begin{array}{c}\text { Artery } \\
\text { (left, right) }\end{array}$ \\
\hline Abdominal aorta & 27 & $51(25,26)$ & $52(26,26)$ & 20 & $40(20,20)$ & $43(23,20)$ & 47 & $91(45,46)$ & $95(49,46)$ \\
\hline Renal artery & 2 & $2(2,0)$ & $2(2,0)$ & 3 & $3(2,1)$ & $3(2,1)$ & 5 & $5(4,1)$ & $5(4,1)$ \\
\hline Other & Not observed & Not observed & Not observed & 2 & $2(0,2)$ & $2(0,2)$ & 2 & $2(0,2)$ & $2(0,2)$ \\
\hline
\end{tabular}

Table 2. Description of the gonadal artery with a single common trunk in the published literature

\begin{tabular}{|c|c|c|c|c|}
\hline References & Sex & Origin & Side & Remark \\
\hline Rusu (2006) [15] & Male & Abdominal aorta & Left & $\begin{array}{l}\text { Doubled right testicular arteries arose from the abdominal aorta and renal artery } \\
\text { Single common trunk run arching with respect to the left renal vein }\end{array}$ \\
\hline Tanyeli et al. 2006 [17] & Male & Renal artery & Right & Normal course of a left testicular artery \\
\hline Kotian et al. 2016 [6] & Male & Abdominal aorta & Left & Normal course of a right testicular artery \\
\hline Mao and Li 2017 [10] & Male & Abdominal aorta & Right & Right testicular vein passed through the hiatus of the right testicular artery \\
\hline First cadaver & Male & Abdominal aorta & Right & $\begin{array}{l}\text { Normal course of a left testicular artery } \\
\text { Lateral right testicular artery run arching with respect to the right renal vein } \\
\text { Inferior vena cava passed through the hiatus of the right testicular artery }\end{array}$ \\
\hline Second cadaver & Male & Abdominal aorta & Right & Normal course of a left testicular artery \\
\hline
\end{tabular}

RTA, mRTA, IRTA, and LTA had an external diameter of approximately $2.4 \mathrm{~mm}, 1.1 \mathrm{~mm}, 1.3 \mathrm{~mm}$, and $1.9 \mathrm{~mm}$, respectively (Fig. 2). The other abdominal arteries displayed normal courses.

The details of the origin of all GAs are shown in Table 1. The details for men and women are shown separately in Table 1 because the gonadal arteries are different in testicular and ovarian arteries. Given that the testicular artery with a single common trunk originates from the $A A$ and then branches into two, it was included in the group branching from the $A A$ in this study. In addition, the single common trunk was included in the ratio of origin as one artery. The GA, testicular artery, and ovarian artery arose from the $A A$ in $93.1 \%, 96.3 \%$, and $89.6 \%$ of cadavers, respectively, and from the renal artery in $4.9 \%, 3.7 \%$, and $6.3 \%$ of cadavers, respectively.

As shown in Table 2, all previous reports of the single common trunk were from case reports of men. There were two testicular arteries with a single common trunk on the right side in the specimens of this study. Therefore, the incidence rate of the single common trunk is $3.7 \%$ of cadavers, $2.0 \%$ of sides, and $2.0 \%$ of arteries in the GA. Moreover, the incidence rate of a single common trunk is $6.9 \%$ of cadavers, $3.8 \%$ of sides, and $3.7 \%$ of arteries in the testicular artery.

\section{DISCUSSION}

Herein, we have described variations at the origin of the GAs in 54 cadavers. In addition, we examined the single common trunk in two cadavers and correlated our observations with related literature. Moreover, we discuss the embryological hypothesis of GAs with a single common trunk.

The GA, testicular, and ovarian arteries arose from the $A A$ in $77.7 \%$ of arteries, $76.5 \%$ of arteries, and $79.3 \%$ of arteries, respectively, and from the renal artery in $21.8 \%$ of arteries, $22.6 \%$ of arteries, and $20.7 \%$ of arteries, respectively $[8,9]$. Our data show that the percentage of vessels arising from the $A A$ was higher than those arising from the renal artery. This result may be due to the limited number of cases. Double testicular arteries existed in $1.6 \%$ and $1.4 \%$ of the right and left sides, respectively. They originated from the same artery in 2 of 8 and 3 of 7 cases at the right and left sides, respectively, and originated from different arteries in 6 of 8 and 4 of 7 cases at the right and left sides, respectively $[8,9]$. In another report [12], double testicular arteries were present in $25 / 1,167(2.14 \%)$ cases on the left side; of these, 14 had both arteries arising from the AA (1.19\%), 9 originated from the AA $(0.77 \%)$. The other one had double testicular arteries originating from arteries other than the $A A$, and 2 had both arteries originating 

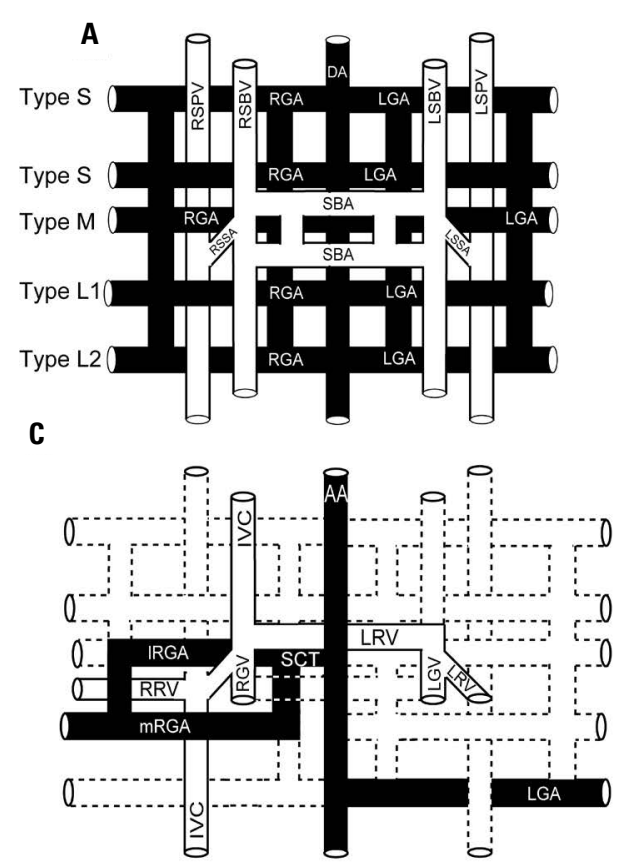

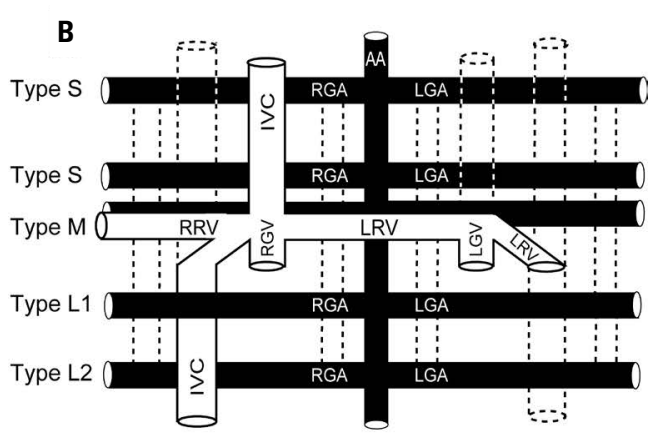

D

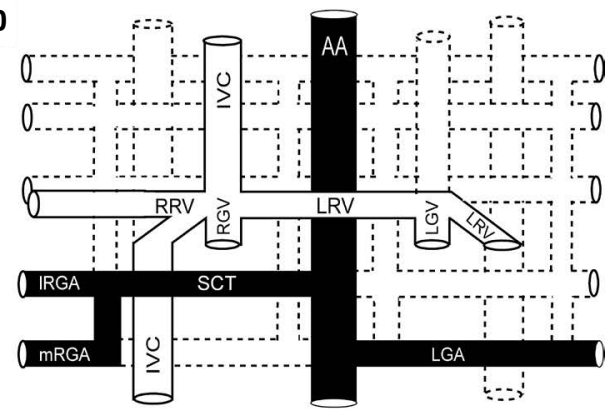

Figure 3. The proposed scheme of the formation of the single common trunk (SCT) of the right testicular artery (modified from the figure of Terayama et al. [18]'s report); A, B. Anastomosis of the right supracardinal vein (RSPV) and right sub-cardinal vein (RSBV) is important to determine the course of the right gonadal artery (RGA); C. Proposed developmental anatomy of the first cadaver (Fig. 1); D. Proposed developmental anatomy of the second cadaver (Fig. 2). Right gonadal arteries (GAs) arising from the dorsal aorta (DA) pass dorsally to the RSPV or pass between the RSBV and RSPV: type $S$ of RGA pass dorsally to IVC and type $M$ and type $L$ of RGA pass dorsally or ventrally to inferior vena cava (IVC); AA — abdominal aorta; mRGA — medial right gonadal artery; LGA — left gonadal artery; LGV — left gonadal vein; LRV — left renal vein; LSBV — left sub-cardinal vein; LSPV — left supracardinal vein; LSSA — left sub-supracardinal anastomosis; IRGA — lateral right gonadal artery; RGV — right gonadal vein; RSSA — right sub-supracardinal anastomosis; RRV — right renal vein; SBA — sub-cardinal anastomosis.

from the vessels other than the AA $(0.17 \%$, both from the RA). On the right side, 19/1,229 cases showed a double testicular artery (1.54\%), 9 had both arteries arising from the $\mathrm{AA}(0.77 \%)$, and 10 had both arteries originating from vessels other than the $A A(0.81 \%)$. It is unclear whether the single common trunk can be included in multiple arteries or single arteries, and the original ratio may be masked. Therefore, a detailed ratio of single common trunk structures of the study specimens is useful for classifying the arteries.

The arterial running pattern of the single common trunk is one of the two types: type A divides into the right and left sides of GAs on the different side and type $B$ divides into the medial and lateral sides of GAs on the same side. The single common trunk structure of type $A$ is reported "in $<0.1 \%$ of cases a common trunk originated from the $A A$, which later divided into right and left testicular arteries" by statistical processing of the previous literature [7]. However, the details of type $B$ are unknown. In this study, the incidence rate was $3.7 \%$ of cadavers and $6.9 \%$ of bodies, $2.0 \%$ of sides and $3.8 \%$ of sides, and $2.0 \%$ of arteries and $3.7 \%$ of arteries in the GA and testicular artery, respectively. Moreover, all single common trunks of type $B$ were present in men, which was consistent with previous literature $[6,10,15,17]$. However, it is unclear whether the single common trunk only exists in men. The testis may be associated with longer migration than the ovary. At any rate, to the best of our knowledge, this report is the first detailed study of the single common trunk of type B.

As shown in Table 2, the RTA with a single common trunk was observed in the cadaveric specimens of this study; however, the embryological significance of the presence of these varieties of the right GAs remains unclear. In general, the variations in the origin, course, and branching out of the right GAs are attributed to the development of the IVC. Only Terayama et al. [18] reported on the development of the RTA and IVC system together. Several successive networks participate in the formation of the IVC through the initial appearance of complex capillary plexuses and preferential utilisation and expansion or regression of individual channels within the plexus $[2,18]$. The sub-cardinal veins arise in association with the ventral margin of the mesonephros and form 
the sub-cardinal anastomosis, which is the origin of the gonadal and renal veins $[4,18]$. Thereafter, the supracardinal veins appear dorsal to the sub-cardinal veins, and the supracardinal and sub-cardinal veins anastomose (Fig. 3A). The sub-cardinal and sub-supracardinal anastomoses, as well as the sub-cardinal and supracardinal veins, are important for the development of the GAs, gonadal veins, renal veins, and IVC (Fig. 3B) [3, 14, 18].

As shown in Figure 3 , we assumed that the right gonadal arteries (RGAs) arise from the dorsal arteries and then pass either dorsally to both the sub-cardinal and supracardinal veins or dorsally to the sub-cardinal veins and ventrally to the supracardinal veins (Fig. 3A). Moreover, several previous reports also showed that the lateral artery of the dorsal aorta, including the right GA, had a longitudinal anastomosis (Fig. 3A) $[13,16]$. Terayama et al. [18] reported that the course of the GA is of one of the four subtypes based on the relationship to the left renal vein and the IVC, of superior $(\mathrm{S})$, middle $(\mathrm{M})$ and lower types $(\mathrm{L} 1=$ ventrally, $\mathrm{L2}=$ dorsal of IVC). Type $S$ ultimately passes dorsally to the IVC (Fig. 3B). Type L can be divided into types $L 1$ and $L 2$ during the development of the IVC (Fig. 3B). Finally, one or two of types S, M, L1, and L2 become the remaining RGA (Fig. 3B). In the first case, it is assumed that a longitudinal anastomosis with type L1 remained after type $\mathrm{M}$ passed through the dorsal side of the IVC (Fig. 3C). It is also presumed that in the second case, a longitudinal anastomosis with type L2 remained after type L1 passed through the ventral side of the IVC (Fig. 3D). The hypotheses of type $S, M$ and $L$ embryology, including the longitudinal anastomosis, are useful in discussing the development of the right $\mathrm{GA}$, including the IVC. The left GA does not need to consider IVC, so either the grid-like vascular network remains partially or not.

Part of the discussion explains the embryological hypothesis of GA based on observations and past literature. Therefore, the limitation of this research is that it has not been proved experimentally.

\section{CONCLUSIONS}

Our findings revealed that the GA, testicular artery, and ovarian artery arose from the AA in $93.1 \%$, $96.3 \%$, and $89.6 \%$ of cadavers, and from the renal artery in $4.9 \%, 3.7 \%$, and $6.3 \%$ of cadavers, respectively. A single common trunk was found in $3.7 \%$ of cadavers, $2.0 \%$ of sides, and $2.0 \%$ of arteries in the
GA and in $6.9 \%$ of cadavers, $3.8 \%$ of sides, and $3.7 \%$ of arteries in the testicular artery. Varying morphological anomalies of GAs may be clinical significant while considering the influence of the blood flow from the gonadal glands and the development of new operative techniques (especially in the laparoscopic surgery field) within the abdominal cavity [13]. The knowledge of these common variations can serve as a reference for avoiding clinical complications, especially during surgery in this region.

\section{Ethical approval and informed consent}

The ethics committee of the Tokai University School of Medicine (No. 17R-309) approved this study.

All the cadavers were donors who had provided direct and informed consent for their bodies to be used for teaching or research projects at the Department of Anatomy.

\section{Acknowledgements}

The authors thank Ms. Kyoko Endo and Ms. Yuko Furuya (both from Tokai University School of Medicine, Kanagawa, Japan) for the excellent secretarial support and Dr. Tsutomu Sato and Dr. Satoshi Kawakami (both from Tokai University School of Medicine, Kanagawa, Japan) for advice. We would like to thank Editage for English language editing.

\section{Funding}

This work was supported by a Grant-in-Aid for Scientific Research (JSPS KAKENHI Grant Numbers 18K10031). The funders had no role in study design, data collection and analysis, decision to publish, or preparation of the manuscript.

\section{REFERENCES}

1. Ciçekcibaşi $A E$, Salbacak $A$, Seker $M$, et al. The origin of gonadal arteries in human fetuses: anatomical variations. Ann Anat. 2002; 184(3): 275-279, doi: 10.1016/S09409602(02)80126-1, indexed in Pubmed: 12056759.

2. Gray SW, Skandala JE. The superior and inferior venae cavae. In: Skandala JE (ed.). Embryology for surgeons. Saunders Company, Philadelphia 1972: 862-876.

3. Itoh M, Moriyama $\mathrm{H}$, Tokunaga $\mathrm{Y}$, et al. Embryological consideration of drainage of the left testicular vein into the ipsilateral renal vein: analysis of cases of a double inferior vena cava. Int J Androl. 2001; 24(3): 142-152, doi: 10.1046/j.13652605.2001.00286.x, indexed in Pubmed: 11380703.

4. Jordan $\mathrm{HE}$, Kindred JA. The inferior vena cava. In: $\mathrm{HE}$ Jordan, JA Kindred (ed.). Textbook of embryology. Appleton-Century, New York 1932: 220-235. 
5. Kayalvizhi I, Narayan RK, Kumar P. Anatomical variations of testicular artery: a review. Folia Morphol. 2017; 76(4): 541-550, doi: 10.5603/FM.a2017.0035, indexed in Pubmed: 28394009.

6. Kotian SR, Pandey AK, Padmashali S, et al. A cadaveric study of the testicular artery and its clinical significance. J Vasc Bras. 2016; 15(4): 280-286, doi: 10.1590/16775449.007516, indexed in Pubmed: 29930605.

7. Lippert $\mathrm{H}$, Pabst R. Arterial variations in man: classification and frequency. 1st Ed. J F Bergman Verlag, Munich 1985.

8. Machnicki A, Grzybiak M. Variations in testicular arteries in fetuses and adults. Folia Morphol. 1997; 56(4): 277-285, indexed in Pubmed: 9635363.

9. Machnicki A, Grzybiak M. Variations in ovarian arteries in fetuses and adults. Folia Morphol. 1999; 58(2): 115-125, indexed in Pubmed: 10598404.

10. Mao QH, Li J. Double right testicular arteries passing through the hiatus in the trifurcated testicular vein. Indian J Surg. 2017; 79(1): 73-74, doi: 10.1007/s12262-0161576-9, indexed in Pubmed: 28331273.

11. Moore KL, Dalley AF. Clinically oriented anatomy, 4th ed. Lippincott Williams and Wilkins, Philadelphia 1999.

12. Nallikuzhy TJ, Rajasekhar SS, Malik S, et al. Variations of the testicular artery and vein: a meta-analysis with proposed classification. Clin Anat. 2018; 31(6): 854-869, doi: 10.1002/ca.23204, indexed in Pubmed: 29737575.

13. Onderoğlu $S$, Yüksel $M$, Arik Z. Unusual branching and course of the testicular artery. Ann Anat. 1993; 175(6): 541-544, doi: 10.1016/s0940-9602(11)80219-0, indexed in Pubmed: 8297041.

14. Pansky B. Development of the venous system. In: Pansky B (ed). Review of medical embryology. Macmillan Publishing, New York 1932: 328-329.

15. Rusu MC. Human bilateral doubled renal and testicular arteries with a left testicular arterial arch around the left renal vein. Rom J Morphol Embryol. 2006; 47(2): 197-200, indexed in Pubmed: 17106531.

16. Shinohara $H$, Nakatani T, Fukuo $Y$, et al. Case with a high-positioned origin of the testicular artery. Anat Rec. 1990; 226(2): 264-266, doi: 10.1002/ar.1092260216, indexed in Pubmed: 2301743.

17. Tanyeli E, Uzel M, Soyluoğlu Al. Complex renal vascular variation: a case report. Ann Anat. 2006; 188(5): 455-458, doi: 10.1016/j.aanat.2006.05.013, indexed in Pubmed: 16999210.

18. Terayama H, Yi SQ, Naito M, et al. Right gonadal arteries passing dorsally to the inferior vena cava: embryological hypotheses. Surg Radiol Anat. 2008; 30(8): 657-661, doi: 10.1007/ s00276-008-0378-6, indexed in Pubmed: 18584112. 